The CPD programme for $S A J C H$ is administered by Medical Practice Consulting.

CPD questionnaires must be completed online at www.mpconsulting.co.za

\section{True $(\mathrm{T})$ or false $(\mathrm{F})$ :}

Regarding cranial ultrasound abnormalities in very low birthweight (VLBW) infants

1. More than $50 \%$ of VLBW infants have evidence of intraventricular haemorrhage.

2. In a Johannesburg Academic hospital, $>90 \%$ of VLBW infants have a cranial ultrasound investigation during their initial hospital stay.

3. Cystic periventricular leukomalacia is associated with chorioamnionitis.

Regarding the management of oesophageal strictures (OSs) in children

4. The most common cause of OSs was repair of oesophageal atresia.

5. Mitomycin-c administration aggravated the stricture in all patients.

\section{Regarding dysphagia in neonates admitted to a neonatal intensive} care unit

6. There are three phases of swallowing in normal neonates.

7. Nearly $50 \%$ of neonates investigated for dysphagia had neurological abnormalities.

Nutritional adequacy of menus in registered child care facilities

8. A child care facility (CCF) open for 6 hours/day should provide one-third of a child's daily nutritional requirements.

9. The majority of CCFs provided the required protein content in the diet.

10. None of the CCFs investigated provided the required calcium content in the diet.
Regarding treatment of severely malnourished children

11.Among HIV-positive children with severe acute malnutrition, those with marasmus have an increased mortality compared to HIV-negative children with severe acute malnutrition (SAM).

12. Good weight gain during recovery from SAM was defined as $>20 \mathrm{~g} / \mathrm{kg} /$ day.

13. HIV infection did not influence the rate of recovery or of weight gain.

\section{Regarding screening for cytomegalovirus (CMV) retinitis}

14. CMV retinitis was found in the majority of referred children with probable systemic CMV infection.

15. CMV polymerase chain reaction was positive in $>80 \%$ of children with probable systemic CMV infection.

16. The majority of children with CMV retinitis were HIV-positive.

\section{Regarding childhood renal disease}

17. Glomerulonephritis was the most common renal disease in children admitted between 1 month and 14 years of age.

18. Proteinuria was present in one-third of children admitted with renal disease.

\section{Regarding neonatal sepsis}

19. Blood, cerebrospinal fluid, and urine are sterile sites in the neonate.

20.Early-onset sepsis is considered to have occurred if the infection is detected within 5 days of neonatal life. 